\title{
Influence of the nozzle head geometry on the energy flux of an atmospheric pressure plasma jet
}

\author{
Thorben Kewitz ${ }^{1,2^{*}}$ (D) Christoph Regula ${ }^{3}$, Maik Fröhlich ${ }^{1,2}$, Jörg Ihde ${ }^{3}$ and Holger Kersten ${ }^{1}$
}

\author{
*Correspondence: thorben.kewitz@ \\ inp-greifswald.de \\ 'Institute of and Applied Physics \\ (IEAP), Kiel University, Leibnizstraße \\ 19, 24098 Kiel, Germany \\ 2Leibniz Institute for Plasma Science \\ and Technology (INP), \\ Felix-Hausdorff-Straße 2, 17489 \\ Greifswald, Germany \\ Full list of author information is \\ available at the end of the article
}

\begin{abstract}
The influence of different nozzle head geometries and, therefore, the variation of the excitation and relaxation volume on the energy flux from an atmospheric pressure plasma jet to a surface have been investigated. Measurements have been performed by passive calorimetric probes under variation of the gas flow through the nozzle. The results show that the geometry of the nozzle head has a significant impact on the resulting energy flux. The relaxation volume affects the dependence of the energy flux on the gas flow. While there is no significant influence of the working gas flow on the energy flux without a relaxation volume, utilizing a relaxation volume leads to a decrease of the energy flux with increasing working gas flow. Within the analyzed parameter range, the energy flux reveals for both nozzle heads a linear dependency on the applied primary voltage.
\end{abstract}

Keywords: Calorimetry, Plasma arc devices, Nozzle head geometry

\section{Springer Open}

\section{Introduction}

Atmospheric pressure plasma treatment has received growing interest in various industrial applications for surface cleaning, activation, functionalization, etching or coating processes during the last decades [1-4]. The processes offer different opportunities and advantages compared to low pressure plasmas, e.g. the lack of maintenance for vacuum equipment or the inline sequential treatment possibilities. The use of atmospheric pressure plasma jets enables the treatment of selected areas without masking. Hence, this technology is very interesting in several applications ranging from automotive industry to medicine $[5,6]$. With such a plethora of applications, the interest in understanding the general interaction mechanisms of an atmospheric pressure plasma jet with a surface in order to improve the processes is high. Even as this field of research is not new, many of the correlated phenomena during the treatment processes are not fully understood up to now [7]. Many open questions are closely correlated to the plasma - surface interactions (especially for layer deposition) since they include chemical as well as physical effects which interact with each other and which strongly influence the results of surface treatment. There are several explanations for the

(c) The Author(s) 2021 licensed Springer on behalf of EPJ. Open Access This article is licensed under a Creative Commons Attribution 4.0 International License, which permits use, sharing, adaptation, distribution and reproduction in any medium or format, as long as you give appropriate credit to the original author(s) and the source, provide a link to the Creative Commons licence, and indicate if changes were made. The images or other third party material in this article are included in the article's Creative Commons licence, unless indicated otherwise in a credit line to the material. If material is not included in the article's Creative Commons licence and your intended use is not permitted by statutory regulation or exceeds the permitted use, you will need to obtain permission directly from the copyright holder. To view a copy of this licence, visit http://creativecommons.org/licenses/by/4.0/. 
various phenomena, but because there are a large number of possible interactions which may vary due to the different treatment parameters it is difficult to identify the dominant quantities. These effects include configuration of electrodes, excitation frequency and voltage, gas composition and gas flow as well as the geometry and dimension of the system [8]. The correlated different characteristics have to be investigated in more detail to develop a more complete theoretical framework.

Meanwhile, for a successful surface treatment some effects have a critical impact on whether plasma treatment can be applied successfully to the substrate material. Among these quantities, the energy flux to the substrate surface plays a crucial role. An excessive energy impact cannot only have a significant impact on the fragmentation and deposition conditions for plasma polymer coatings (chemically and in terms of topography) but can change the characteristic surface properties or result in the destruction of the substrate, respectively [9-11]. In order to correlate the plasma and process parameters to the energy flux and to optimize the processes, related experiments have been performed in this work. The experiments were focused on the influences of the working gas flow and excitation voltage for two different nozzle head geometries of the jet. The gas flow and the applied primary voltage reflect two of the most important jet parameters regarding the excitation energy. Additionally, the nozzle heads provide different excitation and relaxation volumes. The excitation volume is the volume in which the discharge is generated. The ensuing relaxation volume is a confined space, in which the effluent is shielded from the surrounding atmosphere. Some energy flux measurements regarding one of the nozzle head shapes are presented in [12] and for a similar situation to the second nozzle head shape but with a different version of the atmospheric pressure plasma jet system in [13]. Apart from different system versions, which make a comparison of the results difficult, these works focused on other parameters, e.g. the distance between nozzle and substrate.

The energy flux is a crucial process parameter [14-16] and can be measured by a passive calorimetric probe [16]. This diagnostic method has already been successfully applied to various plasma sources at low pressure [17-21], e.g. magnetron, ion beam and RF-plasmas [16, 22-24], and also to atmospheric pressure plasmas [25, 26]. The geometries of the probes vary according to the probe type and the investigated plasma source [27]. The probe design used for the present experiments was adapted to the high energy flux and high gas flow emitted by the plasma jet. The design of the probe is presented in [12] and the data analysis used in this study is the one discussed in [28].

\section{Experimental}

For the experimental investigations we used a commercially available pulsed atmospheric pressure plasma generator type FG 5005 with a HTR 11 transformer in connection to a PFW 10 plasma nozzle from the company Plasmatreat GmbH [29]. Figure 1 shows a schematic drawing of the used plasma jet.

The primary voltage was varied between $250 \mathrm{~V}$ and $450 \mathrm{~V}$ (peak values) resulting in a corresponding primary current of 9 A to 14 A (peak values). The pulse frequency was set to $19 \mathrm{kHz}$ and the duty cycle to $50 \%$. Nitrogen was used as process gas with flow rates in the range of $20 \mathrm{slm}$ to $50 \mathrm{slm}$ adjusted by a mass flow controller by Bronkhorst (F 202 AV). The experimental parameters are summarized in Table 1. 


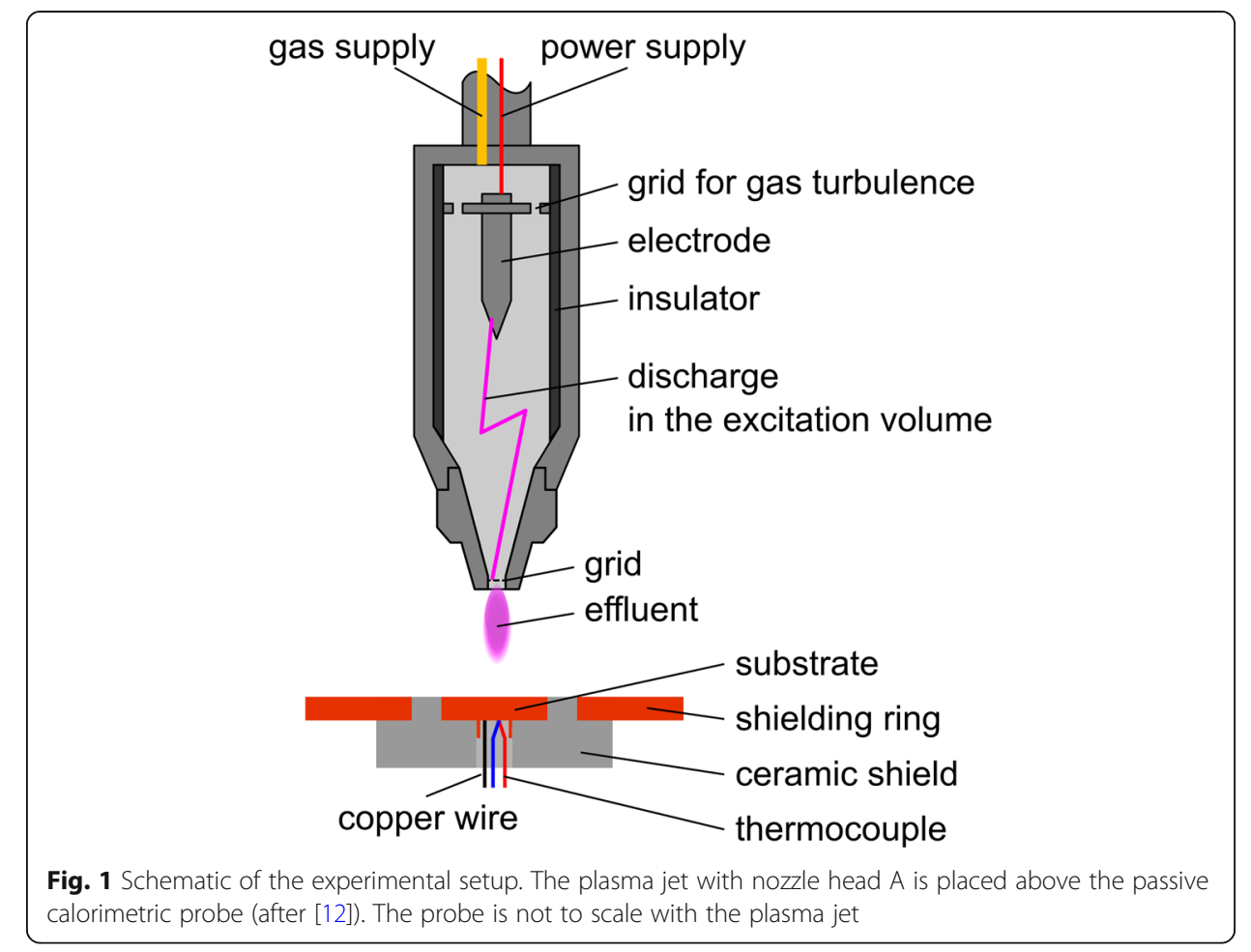

The calorimetric probe used for the energy flux measurements is also shown in Fig. 1. It consists of a $1 \mathrm{~mm}$ thick copper plate with a diameter of $5 \mathrm{~mm}$. A K-type thermocouple and a copper wire are hard soldered to the backside of the probe. The copper plate is surrounded by a ceramic shield made of Macor to protect the thermocouple from direct interaction with the plasma effluent. Furthermore, a second copper plate with a diameter of $40 \mathrm{~mm}$ surrounds the Macor shielding. This second plate imitates the geometry of a large substrate and influences the gas flow accordingly. The temperature signal was sampled with $100 \mathrm{~Hz}$. Before the jet was moved over the calorimetric probe plasma was ignited for $2 \mathrm{~min}$ in a stand-by position to achieve stable discharge conditions. The interaction time between plasma effluent and probe was adapted to the expected energy flux and was in the range of some seconds up to about $40 \mathrm{~s}$. Owing to the different time scales, the calorimetric measurements are integral measurements over many plasma pulses during a treatment cycle.

Two different plasma nozzle head designs, shown in Fig. 2, have been investigated and compared for this study. Nozzle head A made of a Fe-Ni-Cr alloy is consistent with a standardized nozzle head from Plasmatreat for PFW10. The inside is conical and a

Table 1 Overview of the experimental parameters

\begin{tabular}{lll}
\hline Parameter & Range & Standard setting \\
\hline Primary voltage & $250 \mathrm{~V}-450 \mathrm{~V}$ & $400 \mathrm{~V}$ \\
Process gas $\left(\mathrm{N}_{2}\right)$ & $20 \mathrm{slm}-50 \mathrm{slm}$ & $29 \mathrm{slm}$ \\
Pulse frequency & $19 \mathrm{kHz}$ & \\
Duty cycle & $50 \%$ & \\
Sample rate & $100 \mathrm{~Hz}$ & \\
Distance & $4 \mathrm{~mm}-16 \mathrm{~mm}$ & $4 \mathrm{~mm}$ \\
\hline
\end{tabular}




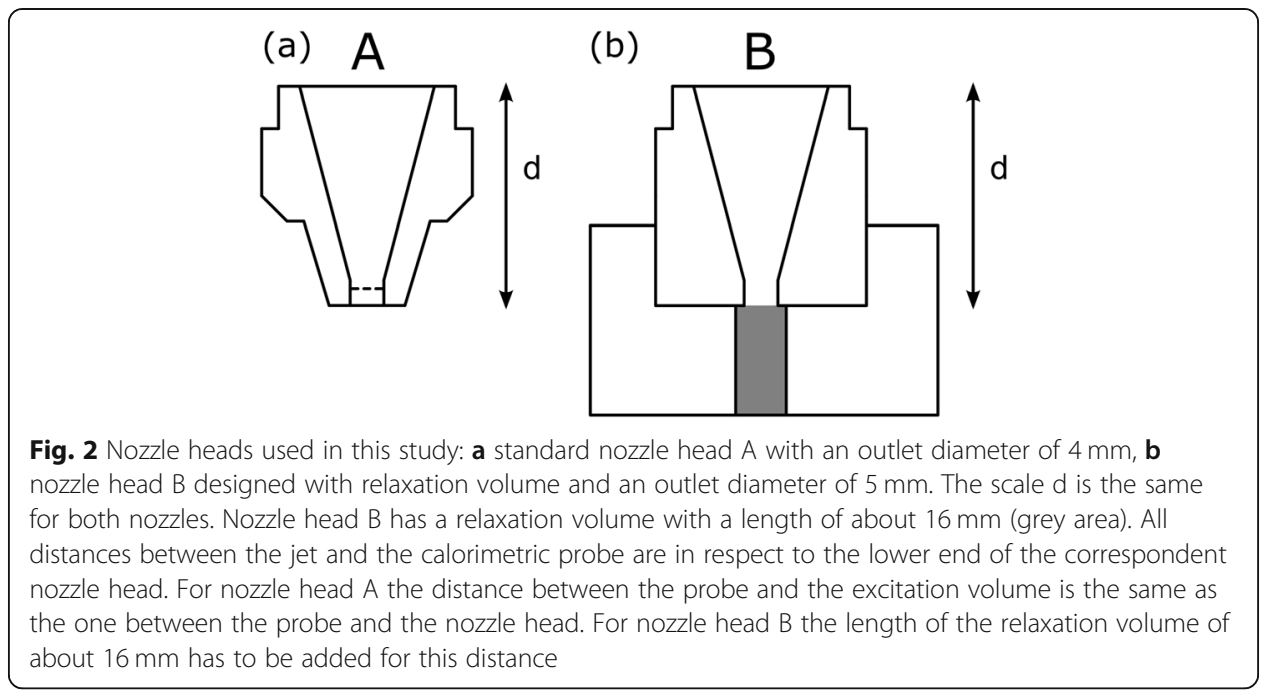

grid is placed near the end of the nozzle exit. The grid limits the expansion of the electrical discharge to the inner volume of the plasma jet resulting in a limited excitation volume. Nozzle head B made of stainless steel was designed for coating applications. In principle, this head has the same inner geometry and the same inner dimensions for the excitation volume as head $\mathrm{A}$, but the grid was omitted and an additional relaxation volume is added directly to the nozzle exit. As the grid no longer limits the discharge length, the effluent can propagate in the adjacent relaxation volume. Hence, the exact excitation and relaxation volumes depend on the discharge length which depends on the discharge parameters, especially, the input power and the gas flow. In general, a distance of $4 \mathrm{~mm}$ between the nozzle and the substrate of the calorimetric probe was chosen which is similar to an industrial working distance for plasma polymer coating processes.

\section{Results and discussion}

The results show a linear dependence of the energy flux on the primary voltage and the primary power within the analyzed parameter range for nozzle head A as well as nozzle head B (compare Fig. 3a and b). This is expected, since an increased amount of energy for a constant gas flow results in a higher plasma density and a higher average temperature, leading to an increased energy flux. The results for nozzle head A are in agreement with those from a similar configuration of plasma jet with nozzle [12]. A comparison between the two nozzle heads emphasizes that the energy flux achieved with nozzle head B is remarkably lower than the energy flux with nozzle head A for the same distance of $4 \mathrm{~mm}$ between nozzle and probe. Here, the same distance relates to the free path in the surrounding atmosphere between the nozzle head and the probe. For both nozzle heads, the fraction reaching the substrate of the probe originates preferably from the center of the flow where the gas has presumably the highest temperature (ref. [12, 30-32]). The relaxation volume of nozzle head B results in an increased time for collisions and, thereby, extended thermalization of the gas due to the added distance between the excitation volume and the probe. This leads to a smaller temperature gradient and less energy transfer through the center of the flow. 
(a)

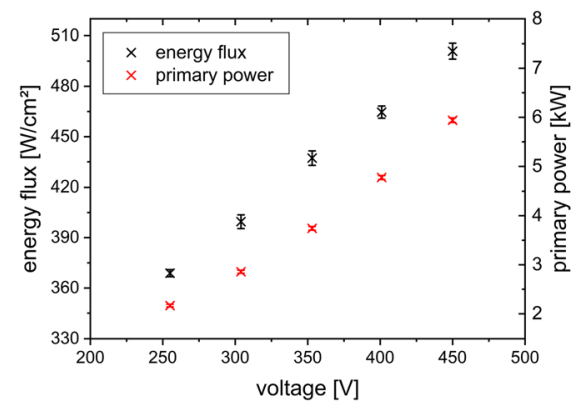

(b)

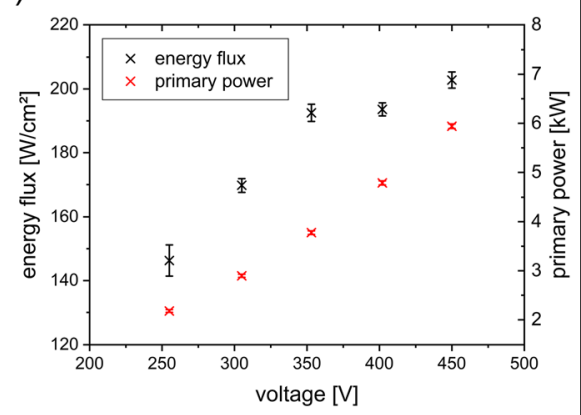

Fig. 3 Influence of the primary voltage on the energy flux (a) for nozzle head A and (b) for nozzle head B. These measurements were performed with a gas flow of $29 \mathrm{slm}$ and at a probe distance of $4 \mathrm{~mm}$ to the nozzle head exit

Additionally, energy is dissipated due to plasma wall collisions resulting in even lower energy fluxes.

Apart from the voltage, the influence of the working gas flow was investigated. The results presented in Fig. 4a and b show different effects. The nozzle head A (Fig. 4a) shows no significant influence on the energy flux by the amount of working gas. Conversely, nozzle head B shows a decreasing energy flux with an increasing amount of gas until $40 \mathrm{slm}$ and stays constant above this value (Fig. 4b). This means, that the geometry of the nozzle head influences the dependence on the gas flow. The decreasing energy flux is attributed to the smaller temperature gradient due to the extended thermalization in nozzle head B. Distributing the available energy to a larger amount of gas leads to reduced gas temperatures and smaller energy fluxes.

The amount of gas can also have an effect on the input energy. The more gas flows through the nozzle, the less ionized and reactive molecules are still within the excitation volume during the next pulse. This leads to a less pre-ionized channel between the electrodes inside the nozzle and, therefore, higher powers for the arc-ignition might be needed.

For nozzle head B the primary current, which is determined by the discharge, stays constant at 11.9 A until $35 \mathrm{slm}$ and rises for higher gas flows up to $12.4 \mathrm{~A}$, leading to increased primary powers. This seems to counteract a further decrease of the energy

(a)

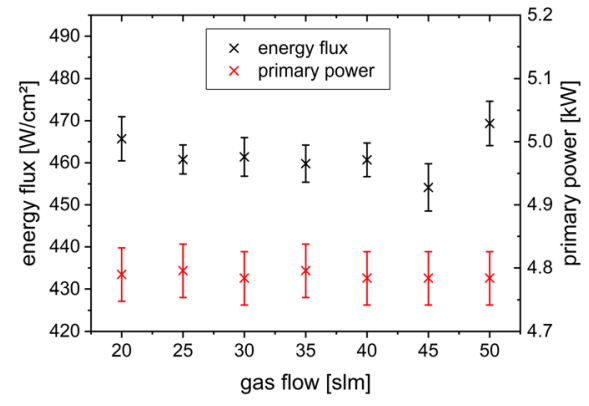

(b)

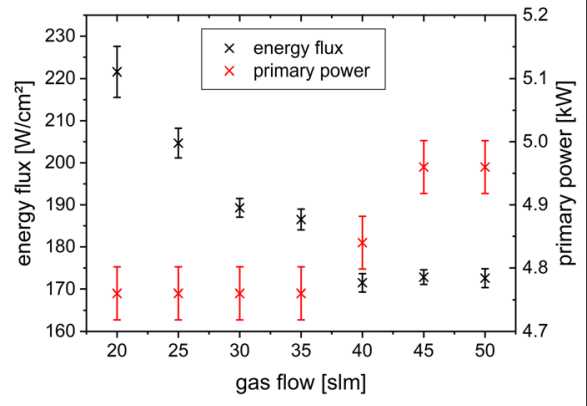

Fig. 4 a Influence of the gas flow on the energy flux (a) for nozzle head A and (b) for nozzle head B. These measurements were performed with a primary voltage of $400 \mathrm{~V}$ and at a probe distance of $4 \mathrm{~mm}$ to the nozzle head exit 
flux due to the larger amounts of gas, resulting in a constant energy flux. As nozzle head B has no grid at the nozzle exit, the higher gas flow might also blow the discharge effluent further out of the nozzle into the relaxation volume during a discharge pulse. Hence, the excitation volume and the discharge length might be extended, which may lead to a higher power for the following pulse as well.

The relaxation volume in nozzle head A is very small in comparison to B. The energy cannot be distributed so efficiently and is concentrated more to the center of the effluent. The primary current is constant at 11.9 A for all gas flows (Fig. 4a). Further, the higher temperatures of the grid reduce the work function, probably leading to an increased stability of the discharge. The constant energy flux implies that the decreased energy to gas ratio is compensated by the increased amount of gas reaching the substrate of the probe due to the higher gas velocities.

Although, the two nozzle geometries show a similar tendency for the dependence of the energy flux on the primary voltage, they differ significantly in their energy fluxes as function of the gas flow. As shown in a previous paper for nozzle head A with a slightly different system (transformer HTR 12: different windings and design; generator FG5001) [12] the general dependence of the energy flux to the probe depends strongly on the distance to the probe. Using nozzle head B increases the distance between the excitation volume and the calorimetric probe due to the additional relaxation volume significantly. In order to investigate if the distance is the reason for the difference in energy flux levels or if the relaxation volume has an additional influence, we performed further measurements at an equal distance of $20 \mathrm{~mm}$ between the excitation volume and the probe. This distance is equivalent to the $4 \mathrm{~mm}$ distance between nozzle head $\mathrm{B}$ and the probe. The results in Fig. 5 are directly compared to those from nozzle head B already shown in Fig. 4b. The energy flux for nozzle head A is considerably lower compared to nozzle head B. Again, the gas flow does not have a significant impact on the energy flux for nozzle head A. We can assume that the energy flux is at the same level in the excitation volume inside the two different nozzles due to the same geometry. The main difference between the two setups is the subdivision of the distance in between the excitation volume and the probe. For nozzle head A the distance is

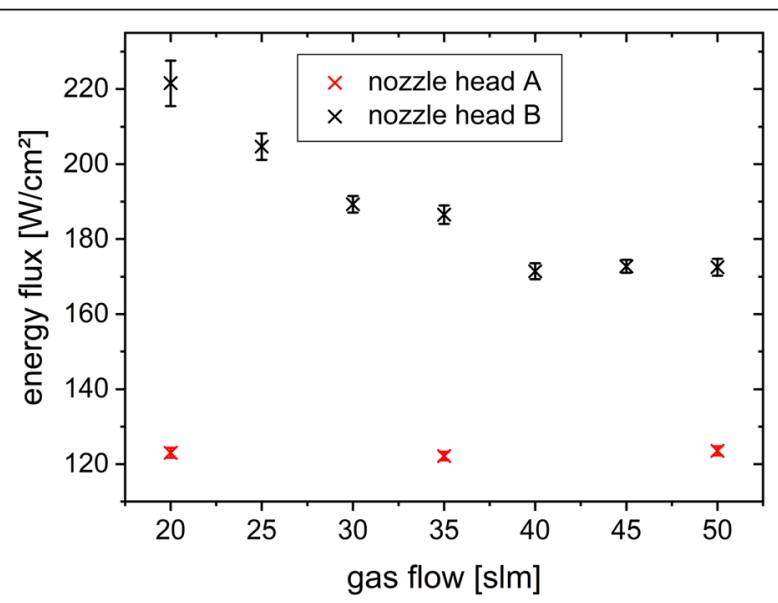

Fig. 5 Influence of the amount of gas on the energy flux from nozzle head A (primary voltage: $400 \mathrm{~V}$ ). The distance of the excitation volume to the probe was set to $20 \mathrm{~mm}$, corresponding to a distance of $4 \mathrm{~mm}$ between probe and nozzle for head B 
characterized by a turbulent flow outside the nozzle with a strong effect of the colliding excited molecules with the neutral molecules of the surrounding atmosphere for 20 $\mathrm{mm}$. This nozzle head seems to form a uniform temperature profile within the flow inside the effluent with decreasing values for higher distances. In comparison, the distance in case of nozzle head B is subdivided in two parts. One part is the relaxation volume with no contact to the outside atmosphere $(16 \mathrm{~mm})$, where only the collisions between the species and with the wall can thermalize the energy distribution and lead to losses. The following part is characterized by a turbulent flow of the species outside the nozzle $(4 \mathrm{~mm})$. While there are losses to the wall, they are small compared to the losses to the surrounding atmosphere, since the wall heats up over time and, therefore, limits the cooling effect along the distance to the probe. This leads to huge differences in the energy flux to the surface showing that the relaxation volume has an additional effect to the added distance.

As a main result our data show, that not only the distance between the excitation volume and the surface is important for the absolute energy flux, but also the manner in which the space in between the excitation volume and the surface is connected to the outer atmosphere.

\section{Conclusion}

It was demonstrated that the energy flux onto the treated material from the presented atmospheric pressure plasma jet can be modified by the design of the nozzle head, the amount of working gas and the primary voltage. The results clearly indicate that the design of the nozzle head not only has a big influence on the energy flux but also on the dependence of the gas flow on the energy flux. While the amount of working gas has almost no influence for the investigated set of parameters for nozzle head A without any relaxation volume, the energy flux changes significantly using nozzle head B with a relaxation volume. Meanwhile, the influence of the primary voltage is comparable for both nozzle heads. It was also shown, that the energy flux is decreased by the relaxation volume due to losses at the wall and thermalization of the gas compared for an equal distance between the nozzle head and the probe. If, conversely, the distance between the excitation volume and the probe is equal, the relaxation volume leads to higher energy fluxes. The reason is that the losses within this volume are small compared to the losses due to gas expansion and mixture with the surrounding atmosphere. Therefore, such a relaxation volume offers the opportunity to treat a substrate with higher energy fluxes at greater distances. These results are of special interest for the adjustment of the energy in the plasma at certain distances from the excitation volumes in order to control the precursor fragmentation. They are further important for the design of new nozzle heads for thermally sensitive substrates. With our findings it is possible to design nozzle heads with tailored energy fluxes. 


\title{
Funding
}

Part of this work was funded by the German Federal Ministry of Education and Research under Project FKZ: 13 N12220 and 13 N12217. Open Access funding enabled and organized by Projekt DEAL.

\section{Availability of data and materials}

The datasets used and/or analyzed during the current study are available from the corresponding author on request.

\section{Competing interests}

The authors declare that they have no competing interests.

\begin{abstract}
Author details
${ }^{1}$ Institute of and Applied Physics (IEAP), Kiel University, Leibnizstraße 19, 24098 Kiel, Germany. ${ }^{2}$ Leibniz Institute for Plasma Science and Technology (INP), Felix-Hausdorff-Straße 2, 17489 Greifswald, Germany. ${ }^{3}$ Fraunhofer Institute for Manufacturing Technology and Advanced Materials (IFAM), Wiener Straße 12, 28359 Bremen, Germany.
\end{abstract}

Received: 4 May 2020 Accepted: 22 December 2020

Published online: 07 January 2021

\section{References}

1. Noeske M, Degenhardt J, Strudthoff S, Lommatzsch U. Plasma jet treatment of five polymers at atmospheric pressure: surface modifications and the relevance for adhesion. Int J Adhes Adhes. 2004;24(2):171-7.

2. Lommatzsch $U$, Ihde J. Plasma polymerization of HMDSO with an atmospheric pressure plasma jet for corrosion protection of aluminum and low-adhesion surfaces. Plasma Process Polym. 2009;6(10):642-8.

3. Meister J, Arnold T. New process simulation procedure for high-rate plasma jet machining. Plasma Chem Plasma Process. 2010;31(1):91-107.

4. Green MD, Guild FJ, Adams RD. Characterisation and comparison of industrially pre-treated homopolymer polypropylene, HF 135M. Int J Adhes Adhes. 2002;22(1):81-90.

5. Weltmann K-D, et al. The future for plasma science and technology. Plasma Process Polym. 2018;16(1):1800118.

6. Schäfer J, Hofmann T, Holtmannspötter J, Frauenhofer M, von Czarnecki J, Gudladt H-J. Atmospheric-pressure plasma treatment of polyamide 6 composites for bonding with polyurethane. J Adhes Sci Technol. 2015;29(17):1807-19.

7. Shi J, Zhong F, Zhang J, Liu DW, Kong MG. A hypersonic plasma bullet train traveling in an atmospheric dielectricbarrier discharge jet. Phys Plasmas. 2008;15(1):013504.

8. Tendero C, Tixier C, Tristant P, Desmaison J, Leprince P. Atmospheric pressure plasmas: a review. Spectrochim Acta B At Spectrosc. 2006;61(1):2-30,

9. Thornton JA. Influence of apparatus geometry and deposition conditions on the structure and topography of thick sputtered coatings. J Vac Sci Technol. 1974;11(4):666-70.

10. Raballand V, Benedikt J, Hoffmann S, Zimmermann M, von Keudell A. Deposition of silicon dioxide films using an atmospheric pressure microplasma jet. J Appl Phys. 2009;105(8):083304.

11. Jacob W. Surface reactions during growth and erosion of hydrocarbon films. Thin Solid Films. 1998;326(1-2):1-42.

12. Kewitz T, Frohlich M, von Frieling J, Kersten $\mathrm{H}$. Investigation of a commercial atmospheric pressure plasma jet by a newly designed calorimetric probe. IEEE Trans Plasma Sci. 2015;43(5):1769-73.

13. Fröhlich M, Bornholdt S, Regula $\mathrm{C}$, Inde J, Kersten H. Determination of the energy flux of a commercial atmosphericpressure plasma jet for different process gases and distances between nozzle outlet and substrate surface. Contrib Plasma Phys. 2014;54(2):155-61.

14. Ding J, et al. Etching rate characterization of $\mathrm{SiO} 2$ and $\mathrm{Si}$ using ion energy flux and atomic fluorine density in a CF4/O2/ Ar electron cyclotron resonance plasma. J Vac Sci Technol A. 1993;11(4):1283-8.

15. Han JG. Recent progress in thin film processing by magnetron sputtering with plasma diagnostics. J Phys D. 2009;42(4): 043001.

16. Kersten H, Deutsch H, Steffen H, Kroesen GMW, Hippler R. The energy balance at substrate surfaces during plasma processing. Vacuum. 2001;63(3):385-431.

17. Thornton JA. Substrate heating in cylindrical magnetron sputtering sources. Thin Solid Films. 1978;54(1):23-31.

18. Kersten H, Rohde D, Berndt J, Deutsch H, Hippler R. Investigations on the energy influx at plasma processes by means of a simple thermal probe. Thin Solid Films. 2000;377-378:585-91.

19. Steffen $\mathrm{H}$, Kersten $\mathrm{H}$, Wulff $\mathrm{H}$. Investigation of the energy transfer to the substrate during titanium deposition in a hollow cathode arc. J Vac Sci Technol A. 1994;12(5):2780-3.

20. Ellmer K, Mientus R. Calorimetric measurements with a heat flux transducer of the total power influx onto a substrate during magnetron sputtering. Surf Coat Technol. 1999;116-119:1102-6.

21. Gardon R. An instrument for the direct measurement of intense thermal radiation. Rev Sci Instrum. 1953;24(5):366-70.

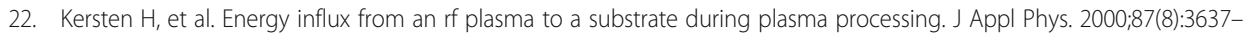
45.

23. Kersten H, Wiese R, Gorbov D, Kapitov A, Scholze F, Neumann H. Characterization of a broad ion beam source by determination of the energy flux. Surf Coat Technol. 2003;174-175:918-21.

24. Rohde D, Pecher P, Kersten H, Jacob W, Hippler R. The energy influx during plasma deposition of amorphous hydrogenated carbon films. Surf Coat Technol. 2002;149(2-3):206-16.

25. Stoffels E, Sladek REJ, Kieft IE, Kersten H, Wiese R. Power outflux from the plasma: an important parameter in surface processing. Plasma Phys Control Fusion. 2004;46(12B):B167-77.

26. Bornholdt $\mathrm{S}$, Wolter $\mathrm{M}$, Kersten $\mathrm{H}$. Characterization of an atmospheric pressure plasma jet for surface modification and thin film deposition. Eur Phys J D. 2010;60(3):653-60.

27. Bornholdt $\mathrm{S}$, Fröhlich $\mathrm{M}$, Kersten $\mathrm{H}$. Calorimetric probes for energy flux measurements in process plasmas. Complex Plasmas. 2014;1:197-234. 
28. Kewitz T, Fröhlich M, Kersten H. Analysis of passive calorimetric probe measurements at high energy influxes. EPJ Techn Instrument. 2017:4(1):1.

29. Plasmatreat GmbH. (Feb 22, 2018). Available: https://www.plasmatreat.de/.

30. Spores R, Pfender E. Flow structure of a turbulent thermal plasma jet. Surf Coat Technol. 1989;37:251-70.

31. Yule AJ. Large-scale structure in the mixing layer of a round jet. J Fluid Mech. 1978;89(3):413-32.

32. Merten C, Regula C, Hartwig A, Ihde J, Wilken R. Track by track: the structure of single tracks of atmospheric pressure plasma polymerized Hexamethyl Disiloxane (HMDSO) analyzed by infrared microscopy. Plasma Process Polym. 2013;10: $60-8$.

\section{Publisher's Note}

Springer Nature remains neutral with regard to jurisdictional claims in published maps and institutional affiliations.

Submit your manuscript to a SpringerOpen ${ }^{\odot}$ journal and benefit from:

- Convenient online submission

- Rigorous peer review

- Open access: articles freely available online

- High visibility within the field

- Retaining the copyright to your article

Submit your next manuscript at $\boldsymbol{\nabla}$ springeropen.com 